\title{
Analysis of IEEE 802.15.4 Beacon-Enabled MAC Protocol
}

\author{
Nga Dinh, Sangsoon Lim
}

Software R\&D Center, Samsung Electronics, Seoul, South Korea

\section{Article Info}

Article history:

Received Mar 5, 2016

Revised Mar 27, 2016

Accepted Apr 10, 2016

\section{Keyword:}

802.15 .4

Analysis

Low Power

WPAN

\begin{abstract}
This paper aims to develop several mathematical models to study medium access control (MAC) protocol in the IEEE 802.15.4 beacon-enabled mode with star topology. In particular, the MAC protocol which employs a slotted carrier-sense multiple access with collision avoidance (CSMA/CA) algorithm used in the contention access period (CAP) of a superframe is modelled. The analysis studies the effectiveness of the CSMA/CA algorithm and provides explicit mathematical expressions for power consumption, access delay, and data frame drop probability. The proposed models precisely follow CSMA/CA algorithm in MAC protocol of beacon-enabled mode and differ from those previously published in the literature as 1) they are derived based on data frame generation rate of end devices, 2) they provide a completed expression for frame access delay, and 3) lowpower states of end devices are considered for power efficiency evaluations. The paper shows how power consumption of end devices is improved on the balance with data frame delay. The validity of the proposed models is confirmed and complemented by extensive simulations.
\end{abstract}

Copyright (c) 2016 Institute of Advanced Engineering and Science. All rights reserved.

\section{Corresponding Author:}

Sangsoon Lim,

Software R\&D Center,

Samsung Electronics,

56, Seongchon-gil, Seocho-gu, Seoul, Korea.

Email:1ssgood80@gmail.com

\section{INTRODUCTION}

Interest in low power wireless protocol has led to work on the IEEE 802.15.4 standard which specifies the physical (PHY) and medium access control (MAC) layers of networks [1]. The standard is developed for personal area networks (PANs) with low power consumption, low complexity, and high reliability which enable PANs to be widely employed in many industrial applications such as E-healthcare, home automation, environmental monitoring, and industrial automation [2]. A PAN is composed of one PAN coordinator and multiple end devices (hereafter denoted as devices) which transmit data frames (hereafter denoted as frames) to the coordinator. Depending on the application requirements, an IEEE 802.15.4 PAN can operate in either of two topologies: star topology and peer-to-peer topology. In the star topology, the communication is established between devices and the PAN coordinator. In peer-to-peer topology, each device is capable of communicating with any other devices within its radio sphere of influence. The IEEE 802.15.4 standard supports two channel access algorithms. A beacon-enabled PAN uses a slotted carrier sense multiple access with collision avoidance (CSMA/CA) algorithm to access radio channel. The slot boundaries of each device are aligned with the slot boundaries of the PAN coordinator. On the other hand, if beacons are not available (non-beacon-enabled PAN), a simple unslotted CSMA/CA algorithm is used.

There has been diverse researches on the IEEE 802.15.4 standard (see [3]-[7],[9],[10],[12]-[16] and references therein). Performance evaluation of the IEEE 802.15.4 MAC protocol has been carried out by simulations for small and low load networks in [4] and for dense networks in [5]. Its throughput and delay were also evaluated by measurements and simulations in [6]. In addition, Wang et al. [7] compared power consumption, throughput, and end-toend delay between slotted and unslotted CSMA/CA algorithms by using 
OPNET simulations. More recently, the authors [8] carried out an extensive analysis based on both simulations and experiments to investigate energy efficiency, scalability, reliability, and timeliness problems of IEEE 802.15.4. MAC parameters were set to increase delivery ratio but with the cost of an increased latency. Furthermore, some studies have analytically evaluated the behavior of slotted CSMA/CA algorithm in IEEE 802.15.4 MAC protocols. In [9], the authors derived the probability distribution of access delay and throughput of IEEE 802.15.4 beacon-enabled PAN with uplink transmission in non-saturation mode. In another study [10], Pollin et al. presented an analytical model for MAC layer and evaluated throughput and energy for slotted CSMA/CA algorithm. The key approximation in the model is that carrier sensing probability is constant and independent. Delay analysis for IEEE 802.15.4 CSMA/CA algorithm with heterogeneous buffered traffic was analyzed in [11]. The authors here used a Markov chain to present CSMA/CA process and M/G/1/K queues to character the buffered condition. Those works [9]-[11] are based on model in [12] and used a Markov chain to describe device states. However, the status of backoff time counter is not Markovian as its value depends on the past history (i.e., how many times a device has attempted to access radio channel and found it busy; after each time, the backoff exponent is increased by one). Throughput and energy were also analyzed in [13] with the assumption that the uniform distribution of backoff counter is geometrical so that the backoff algorithm becomes memoryless. The evaluation of IEEE 802.15.4 MAC protocol is further studied in [14]. The author here proposed a model to describe the probability of packet successful transmission reception and mean delay statistic for both contention access period (CAP) and contention free period (CFP) in a superframe in star and tree topologies. The model used Markov chain assuming that each device always has one frame to transmit. Throughput and delay of unslotted IEEE 802.15.4 were addressed in [15] but with simple network condition: the PAN consists of only one coordinator and one device. More recently, the authors in [16] proposed a novel adaptive duty cycle algorithm which makes maximum use of network traffic for automatically adjusting duty cycle to minimize power consumption.

Power consumption is one of the foremost concerns for the IEEE 802.15.4 connected devices. However, most previous research did not sufficiently study power consumption. In particular, low-power states which can significantly improve power efficiency were not well-studied even the IEEE 802.15.4 has inactive period during which devices to enter low power mode for power saving. This paper therefore considers different power states while modeling power consumption for IEEE 802.15.4 connected devices. Besides power consumption, delay and frame drop probability which would degrade quality of service (QoS) for many applications also need to be considered seriously. For example, in home automation, users expect to operate and monitor their homes remotely at any time. They also need their homes real-time information, for instance, when temperature reaches a certain threshold. Even applications with stringent timing requirements can be solved by using contention free period (CFP) of a superframe, the number of guaranteed time slots (GTSs) in the CFP is limited to seven [14]. Mean delay of a frame was also mentioned in [14] but the model here started from the reception of the beacon frame. In fact, access delay is calculated from the point the frame is generated until the point it is actually sent. Unlike the approach in [14], we propose a complete model for frame access delay. In addition to delay, we propose models for power consumption and frame drop probability while considering the limitations of previous research. The proposed models are derived frame generation rate of devices and thus they are more realistic and novel approach compared to previous studies.

The rest of this paper proceeds as follows. Section II describes the slotted CSMA/CA algorithm in IEEE 802.15.4. Analytical models are presented in Section III. Next, Section IV provides simulation results to validate our models. Finally, Section V concludes our paper.

\section{SLOTTED CSMA/CA ALGORITHM}

According to the IEEE 802.15.4 MAC protocol [1], the access to the channel is managed through a superframe, starting with a beacon frame transmitted by the PAN coordinator. The superframe is divided into 16 equally size slots. Optionally, the superframe can have an active period and an inactive period. Inactive period is used for power saving as devices can switch off during this period. The active portion may consist of a CAP and a CFP, as shown in Figure 1. 


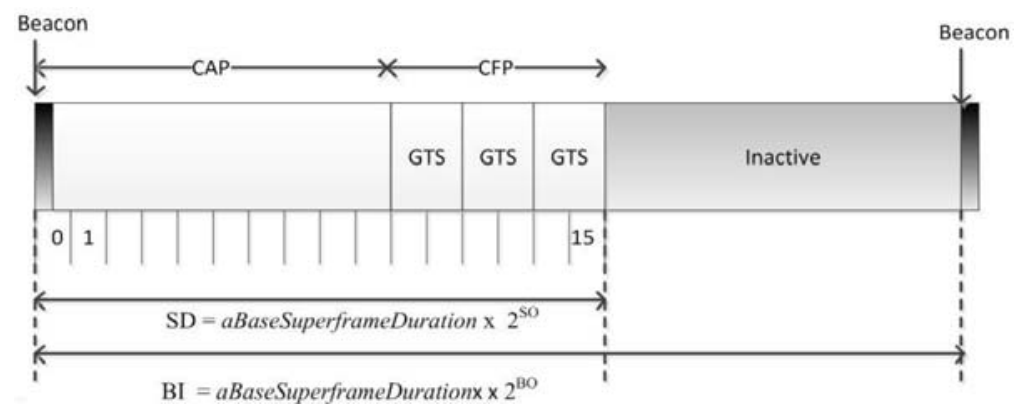

Figure 1. Superframe structure consisting of an active period and an inactive period

Any device wishing to communicate during CAP competes with other devices using a slotted CSMA/CA algorithm. In CFP, GTS portions are dedicated by the PAN coordinator to applications that require specific data bandwidth [1]. The length of the superframe (called the beacon interval, BI) and the length of active period (called superframe duration, SD) are defined as follows [1]: BI = aBaseSuperframeDuration $\times \quad 2^{B O}$ and $\mathrm{SD}=$ aBaseSuperframeDuration $\times 2^{\text {SO }}$ where aBaseSuperframeDuration $=960$ symbols or $15.36 \mathrm{~ms}[14] ; B O$ and $S O$ denote the beacon order and superframe order, respectively. These values are determined by the coordinator and satisfied $2 \leq S O \leq B O \leq$ 14. The ratio of active period to the whole superframe duration, which is equal to $2^{-(B O-S O)}$, is called duty cycle.

CSMA/CA algorithm is used before transmission of a data frame. In slotted CSMA/CA algorithm, the backoff slot boundaries of each device are aligned with the superframe slot boundaries of the coordinator. The beginning of the first back off period of each device is aligned with the beginning of beacon frame transmission. However, transmissions may start on the boundary of backoff slots. Each device maintains three variables for transmission attempt: $N B, C W$, and $B E . N B$ is the number of times CSMA/CA algorithm was required to back off while attempting the current transmission. $C W$ is the number of backoff slots, denoted as $t_{b-s l o t}$, that need to be clear of channel activity before transmission. $N B$ is the backoff exponent related to how many $t_{b-s l o t}$ a device shall wait before attempting to access channel [1].

In beacon-enabled PANs, when a device wishes to transfer data to a coordinator, it first listens for the network beacon. When beacon is found, the device synchronizes to the super frame structure. Then, it transmits data frame to the coordinator using slotted CSMA/CA algorithm, as explained in Figure 2. First, $N B, C W$, and $B E$ are initialized to 0,2 , and $B E_{\text {min }}$, respectively, and device locates the boundary of next backoff period (Step 1). Upon reception of the beacon, device delays for a random number of $\mathrm{t}_{\mathrm{b} \text {-slot }}$ slots in the range from 0 to $2^{B E}-1$ (Step 2). Then, the PHY layer performs clear channel assessment (CCA) (Step 3). The carrier sensing starts on a backoff period boundary. The MAC layer then proceeds, provided that the remaining CSMA/CA algorithm steps including frame transmission and any acknowledgment are completed before the end of a CAP slot. If the MAC layer cannot proceed, it must wait until the start of CAP in next superframe and then repeat again. If the channel is assessed busy (Step 4), the MAC layer increases both $N B$ and $B E$ by one, ensuring that $B E$ is not more than $a M A X B E$, and $C W$ is set to 2 . If $N B$ is less than or equal to macMaxCSMABackoffs, the CSMA/CA algorithm returns Step 2, else it terminates with Transmission Failure status. If the channel is assessed to be free (Step 5), the MAC layer must ensure that the contention window is expired before data transmission. For this, the MAC layer decreases $C W$ by 1 . If $C W$ is greater than 0 , it must go to Step 3, else it starts to transmit on the boundary of the next slot period. 


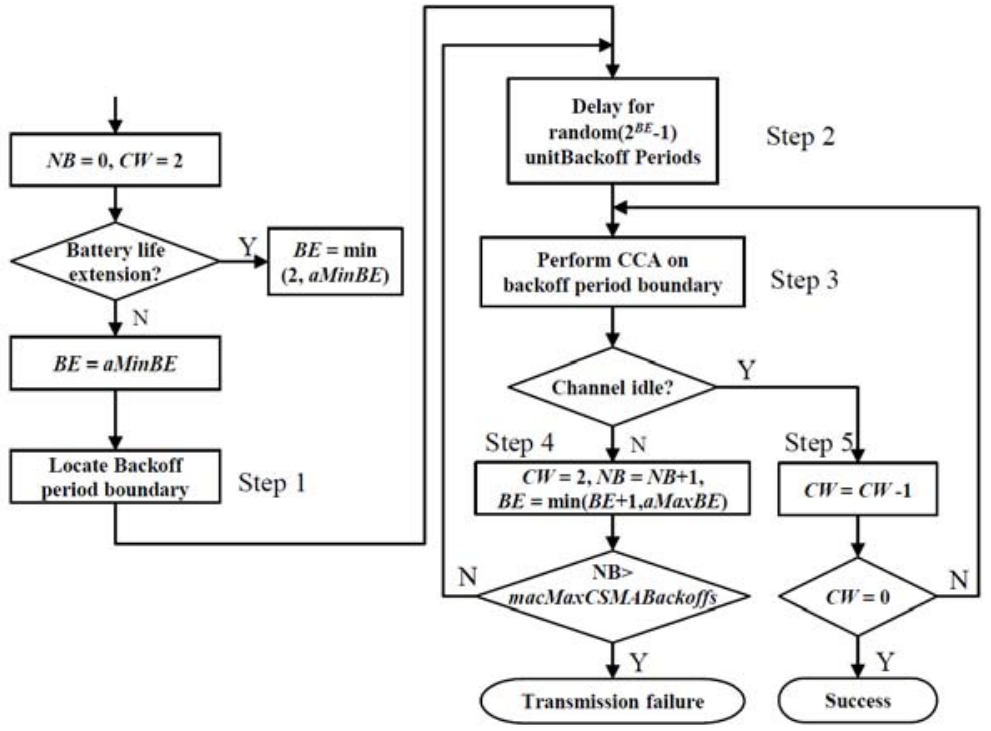

Figure 2. Slotted CSMA/CA Algorithm

\section{ANALYTICAL MODELS}

The analysis is restricted to upstream traffic from devices to the PAN coordinator. We assume that there is one PAN coordinator and two devices in IEEE 802.15.4 star topology. Frame generation rate of each device is assumed to follow Poisson distribution with a rate (number of frames per second). In addition, CAP slot of a superframe is assumed to be long enough so that frame transmission is finished within one CAP slot. In addition, since acknowledgment is optional in the IEEE 802.15.4 standard [1], no acknowledgment is implemented for simplicity. The IEEE 802.15.4 standard limits the number of times that a device tries to attempt current transmission to 3 . This paper therefore set $N B$ to 3 . If a frame cannot be transmitted after three attempts, it will be dropped. Otherwise, the device transmits that frame for a duration $\mathrm{T}_{\mathrm{T}}$. This section derives numerical models for 1) mean access delay of data frames 2) mean power consumption of a device, and 3) data frame drop probability during CAP of superframe.

\subsection{Average Access Delay}

Before sending a frame to the PAN coordinator, the device first waits for the network beacon [1]. We let $\mathrm{T}_{W}$ denote this waiting time. As the authors are aware, most previous works did not consider $\mathrm{T}_{W}$ but $\mathrm{T}_{W}$ can significantly impact overall delay as we will see in Section IV. Access delay refers to the interval the frame is generated up to the point that the frame is actually sent. Only access delay for successfully transmitted frames is considered, i.e., delay caused by dropped frames is ignored. Let $\mathrm{T}_{S}$ denote the duration of a superframe. To calculate $T_{W}$, we divide the superframe into $M$ small subintervals where $M$ is sufficient large. Let $\quad m$ denote the waiting time for beacon frame of a data frame generated during the $m$ th sub-interval, $m$ is given by

$$
\tau_{w}=T_{S}\left(1-\frac{w}{M}\right)
$$

Because Poisson process is memoryless, the probabilities of frame generated during each subinterval are the same. Trivially then, $\mathrm{T}_{W}$ is determined as:

$$
T_{W}=\frac{1}{M} \sum_{W=1}^{M}\left(1-\frac{m}{M}\right)=\frac{T_{2}}{2}-\frac{T_{2}}{2 N}
$$

Since $\lim _{M \rightarrow \infty} \frac{T_{z}}{2 M}=0, T_{W}$ can be simply re-written as:

$$
T_{W}=\frac{T_{z}}{2}
$$

Since the number of attempts to data transmission is limited to 3, we consider three following cases. 
Case 1: A device transmits data after only one attempt, as shown in Figure 3. Let $\mathrm{T}_{1}, \mathrm{~T}^{1}{ }_{B}$, and $\mathrm{T}^{1}{ }_{C C A}$ be the mean access delay, backoff duration, and carrier sensing durations, respectively. At the beginning of a superframe, the device backs off and backoff duration is uniformly distributed from 0 to $2^{B E}-1, \mathrm{~T}^{1}{ }_{B}$ is given by

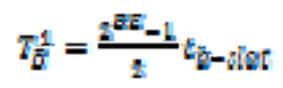

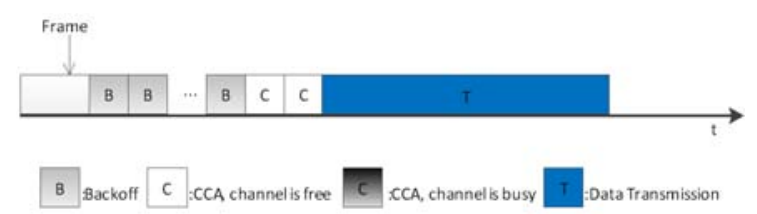

Figure 3. CSMA/CA operation when a device transmits data after only one attempt

Next, PHY layer performs carrier sensing during two consecutive $t_{b-s l o t}$ slots. Since the device transmits data in the first attempt, channel is free during carrier sensing. Let $p$ be the probability that channel is free during one $t_{b-s l o t}$ slot. Since Poisson process is memoryless and backoff period is uniform distribution, $p$ is also the probability that no frame is generated during $t_{b-s l o t}$. According to Poisson process, $p$ is given by

$$
p=\sigma^{-\square t h-s h a s}
$$

Let $P_{1}$ be the probability of case 1 , we then have

$$
P_{1}=p^{2}
$$

The mean access delay of case 1 is therefore determined as

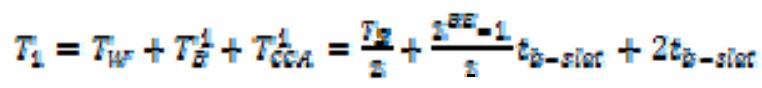

Case 2: A device transmits data after two attempts, as shown in Figure 4. This means that during carrier sensing of the first attempt, the channel is busy either in the first $t_{b-s l o t}$ (subcase 2-1) or in the second $t_{b-}$ slot given that the first $t_{b-s l o t}$ is free (subcase 2-2). In the second attempt, channel is free during two consecutive $t_{b-s l o t}$ of sensing process. These two subcases are the same, except total number of $t_{b-s l o t}$ slots for carrier sensing. Let $\mathrm{P}_{2}^{1}$ denote the probability of subcase $2-1, \mathrm{P}_{2}^{1}$ is given by

$$
P_{2}^{4}=(1-p) p^{2}
$$

Similarly, let $\mathrm{P}_{2}^{2}$ denote the probability of subcase $2-2, \mathrm{P}_{2}{ }_{2}$ is given by

$$
P=(1-p) p^{2}
$$

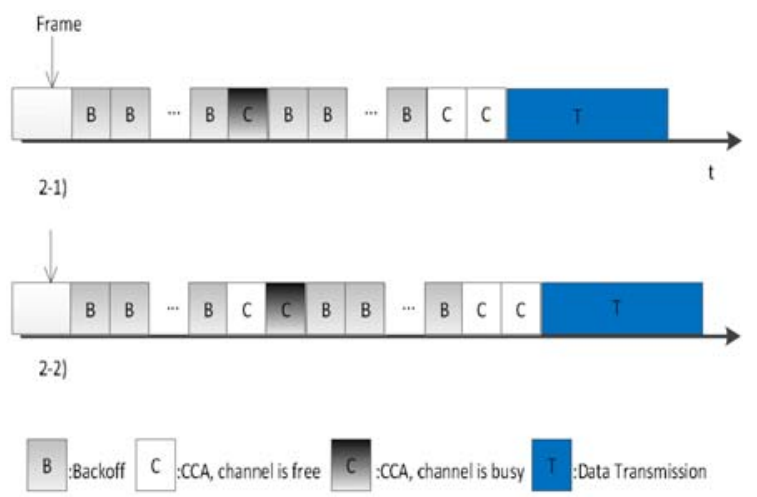

Figure 4. CSMA/CA operation when a device transmits data after two attempts 
In addition, let $\mathrm{T}_{2}^{1}$ and $\mathrm{T}_{2}^{2}$ be the mean access delay of subcase 2-1 and 2-2, respectively. Then $\mathrm{T}^{1}{ }_{2}$ and $\mathrm{T}_{2}^{2}$ are determined as follows:

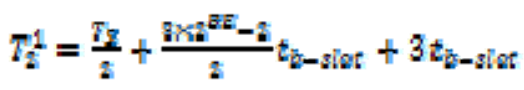

$$
\begin{aligned}
& T_{2}^{2}=\frac{T_{2}}{2}+\frac{9 \sin ^{2 \pi}-2}{2} t_{\bar{\alpha}-\sin t}+4 t_{\bar{\alpha}-\sin t}
\end{aligned}
$$

In (10) and (11), the middle term is mean backoff duration, denoted as $\mathrm{T}^{2}{ }_{B}$, and the last terms are the total carrier sensing durations for subcases $2-i$, denoted as $\mathrm{T}^{i}{ }_{C C A 2}$ with $(i=1,2)$. Case 3: A device transmits data after three attempts. This means that radio channel is busy during carrier sensing in first two attempts but free in the last attempt. Case 3 is further divided into four subcases $3-j(j=1,2,3,4)$, as shown in Fig. 5. These subcases are the same, except total number of $\mathrm{t}_{b \text {-slot }}$ slots for carrier sensing. Let $\mathrm{P}_{3}^{j}$ and $\mathrm{T}_{3}^{j}$ be the probability and mean access delay in subcase $3-j$, we have the followings:

$$
\begin{aligned}
& F_{g}=(1-p)^{2} p^{2}
\end{aligned}
$$

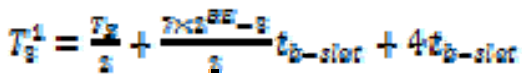

$$
\begin{aligned}
& P_{7}^{2}=(1-p)^{2} p^{2}
\end{aligned}
$$

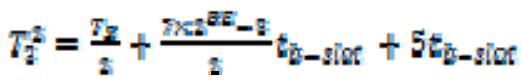

$$
\begin{aligned}
& F^{2}=(1-p)^{2} p^{2}
\end{aligned}
$$

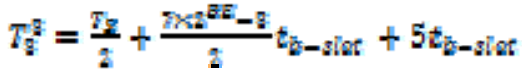

$$
\begin{aligned}
& F_{g}^{4}=(1-p)^{2} p^{4}
\end{aligned}
$$

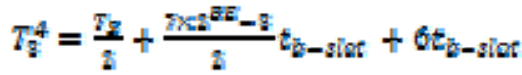

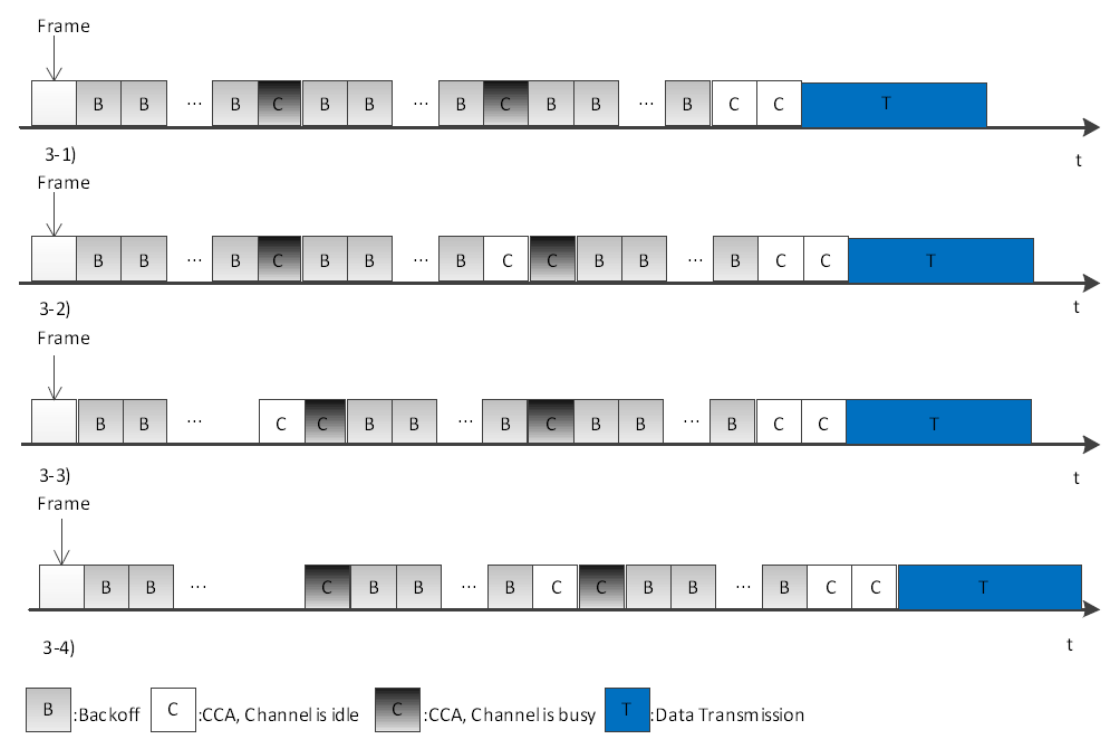

Figure 5. CSMA/CA operation when a device transmits data after three attempts 
In (13), (15), (17), and (19), the middle term is the mean backoff duration, denoted as $\mathrm{T}^{3}{ }_{B}$; the last terms are the total carrier sensing durations for subcases $3-j$ and are denoted as $\mathrm{T}^{j}{ }_{C C A 2}$ with $(j=1,2,3,4)$. Let $\mathrm{T}_{D}$ be the final mean frame access delay. Then, $\mathrm{T}_{D}$ is determined such that:

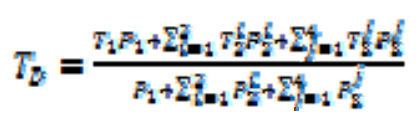

where $\mathrm{T}_{1}, \mathrm{~T}_{2}^{i}, \mathrm{P}_{2}^{i}(\mathrm{i}=1,2), \mathrm{T}_{2}^{j}$, and $\mathrm{P}_{2}^{j}(\mathrm{j}=1,2,3,4)$ are determined by (7)-(19).

\subsection{Power Consumption}

We consider a completed cycle from the point a frame is generated until the point it is completely transmitted. Since in beacon-enable PANs, each device knows when the next beacon frame arrives, we assume that the device is switched off while it is waiting for the beacon frame to reduce power consumption. In addition, we consider the Chipcon 802.15.4 compliant RF transceiver, CC2420 [15]. The Chipcon radio supports the following four states:

- Sleep: when device is waiting for a beacon frame.

- Idle: when device is backing off.

- Receive: when device is doing carrier sensing.

- Transmit: when device is transmitting frame.

Let $\mathrm{W}_{S}, \mathrm{~W}_{I}, \mathrm{~W}_{\mathrm{R}}$, and $\mathrm{W}_{T}$ be the power that device consumes in Sleep, Idle, Receive, and Transmit states, respectively. Then $\mathrm{W}_{S}<\mathrm{W}_{I}<\mathrm{W}_{T}<\mathrm{W}_{R}$ [15]. In addition, let $\mathrm{W}_{1}, \mathrm{~W}_{2}^{i}(i=1,2)$, and $\mathrm{W}_{3}^{j}(j=1,2,3,4)$ be the power consumption of case 1 , subcase $2-i$, subcase $3-j$, respectively. Then $W_{1}, W_{2}^{i}$, and $W_{3}^{j}$ are determined as follows:

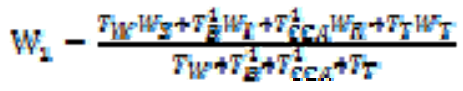

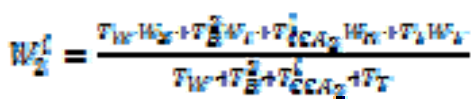

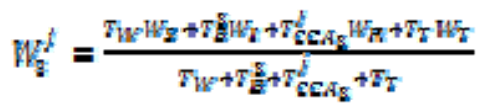

Assume that power and time when the device transitions from one power state to another can be ignored and let $\mathrm{W}$ denote the final power consumption of the device, then $\mathrm{W}$ is approximately expressed as

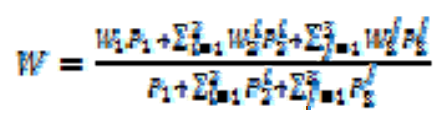

where $\mathrm{W}_{1}, \mathrm{~W}_{2}^{\mathrm{i}}$, and $\mathrm{W}_{3}^{\mathrm{j}}$ are calculated by (21), (22), and (23), respectively. $\mathrm{P}_{2}^{i}$ and $\mathrm{P}_{3}^{j}$ are calculated in subsection III-A.

\subsection{Drop Probability}

Let $\mathrm{P}_{\text {drop }}$ denote the frame drop probability during CAP. A frame is dropped if channel is busy during carrier sensing in all three attempts. $\mathrm{P}_{d r o p}$ is determined in the following way:

$$
R_{\text {drop }}=\left(1-p^{2}\right)^{2}
$$

Intuitively, higher data frame generation rate or larger number of end devices in the network would increase collision probability. As a result, there will be an increase in frame drop probability.

\section{PERFORMANCE EVALUATION}

To supplement the analytical results and to determine the performance in terms of power consumption, access delay, and frame drop probability, $\mathrm{C}$ programming is used to simulate upstream traffic from devices to the PAN coordinator. This section also compares mathematical results obtained from analysis in section III with simulation results obtained from $\mathrm{C}$ programming. Frame generation rates are modeled as a Poisson process; the performances of other traffic models are currently being investigated. 
Since a data frame has 6 bytes for synchronization and PHY headers, 0-127 bytes for payload, the frame size varies from 6 to 133 bytes [1]. In addition, the data rate of the IEEE 802.15 .4 is $20 \mathrm{kbps}, 40 \mathrm{kbps}$, and 250 $\mathrm{kbps}, \lambda$ can be varied in the range $[19 ; 5208]$ (frames/second). Let $\mathrm{D}$ be the number of $\mathrm{t}_{b-\text { slot }}$ slots that each frame occupies while being transmitted, then $\mathrm{D}$ is an integer in the range [2],[13] depending on the minimum and maximum possible frame size [1],[12]. In our simulation, $\mathrm{D}$ is fixed at 3 resulting $\mathrm{T}_{T}$, which is equal to $\mathrm{D}$. $\mathrm{t}_{b \text {-slot, }}$ is $0.96 \mathrm{~ms}$. The superframe length is defined by the IEEE 802.15 .4 standard as aBaseSuperframeDuration $\times 2^{B O}$ where aBaseSuperframeDuration is $15.36 \mathrm{~ms}$ and $0<B O<14$. Therefore our simulation, BI is varied from $100 \mathrm{~ms}$ to $1000 \mathrm{~ms}$. The simulations run for 1,000,000 time units and the results are the mean values from 100 different runs with 100 different seed values.

Figure 6 illustrates how mean access delay $T_{D}$ depends on superframe length $T_{S}$ when $\lambda$ is fixed at 10 (frames/second). In this figure, $\mathrm{T}_{D}$ seems to be linearly increased with $\mathrm{T}_{S}$. This can be explained as follows: from equation (20) and related equations for mean access delay, we observe that $\mathrm{T}_{D}$ is composed of $\mathrm{T}_{S} / 2$ which is much larger than remaining terms because a superframe is much longer than a CAP slot and backoff duration. In fact, from both simulation and numerical analysis, we see that $\mathrm{T}_{D}$ slightly increases with $\lambda$. That is because a higher $\lambda$ increases the probability that the radio channel is busy during carrier sensing, which makes the device try other attempts to access radio channel. Thus, access delay is increased. However, since $\lambda$ just has a small impact on $\mathrm{T}_{D}$, we omit the effects of $\lambda$ in the figure.

Figure 7 evaluates power consumption of a device while lowpower states are employed. The parameters are chosen based on [15] as follows: $\mathrm{W}_{S}=144 \mathrm{~mW}, \mathrm{~W}_{I}=712 \mu \mathrm{W}, \mathrm{W}_{R}=35.28 \mathrm{~mW}$. $\mathrm{W}_{T}$ can be changed based on setting, we therefore choose transmit mode at $0 \mathrm{dBm}$ or $\mathrm{W}_{T}=1 \mathrm{~mW}$. The figure illustrates how power consumption depends on $\mathrm{T}_{S}$ when $\lambda$ is fixed 10 and 100 (frames/second). As $\mathrm{T}_{S}$ increases, the power consumption dynamically decreases. The reason is that a higher $\mathrm{T}_{S}$ makes a device sleep for a longer time. In fact, this sleep time is dominant in one operation cycle of the device. The figure also shows that employing lowpower states significantly improves power efficiency. Power consumption in the worst case of our simulation is lower than that in Idle state and is much more lower than power consumption in Transmit or Receive states. Furthermore, it can be inferred from Figure 6 and Figure 7 that power consumption and access delay have a tradeoff relationship. Increased superframe length results in a better power saving but simultaneously results in a worse delay performance. Therefore, superframe duration can be appropriately chosen on the balance between power consumption and access delay.

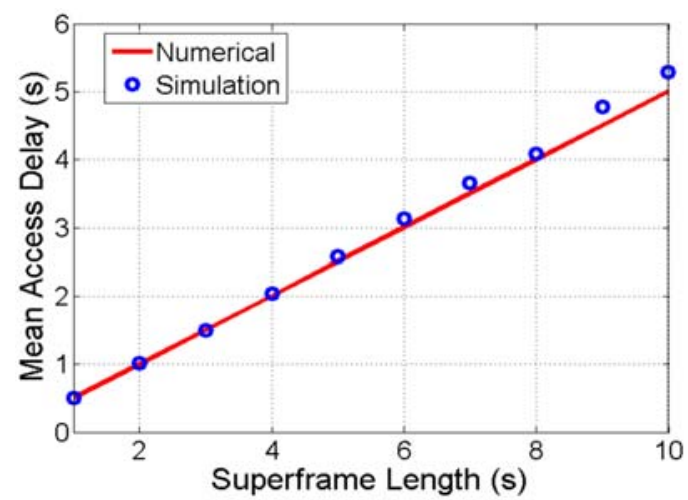

Figure 6. Mean access delay $\mathrm{T}_{D}$ with respect to the superframe length $\mathrm{T}_{S}$

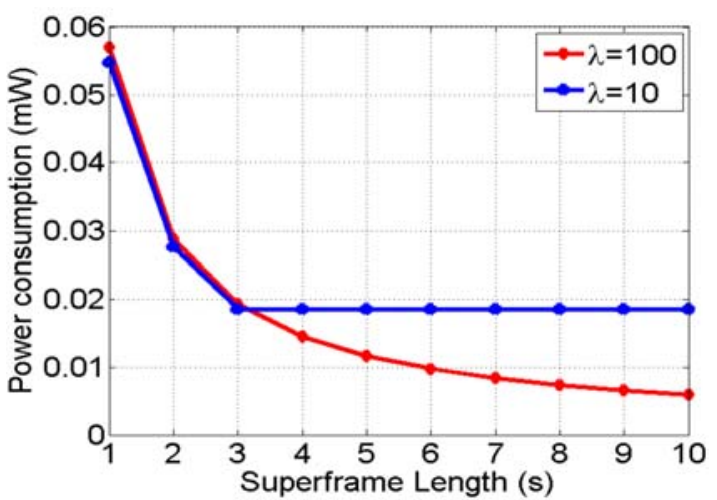

Figure 7. Power consumption $\mathrm{W}$ with respect to superframe length $\mathrm{T}_{S}$

Figure 8 illustrates how frame drop probability Pdrop depends on $\lambda$. As observed from the figure, as $\lambda$ increases, $P_{d r o p}$ dynamically increases. That is because increase in $\lambda$ results in higher probability that the radio channel is busy during carrier sensing. Since the number of attempts to current transmission is limited to three, the frame drop probability is increased. In addition, it can be inferred from Figure 8 that $\lambda$ has strong impact on frame drop probability; however, it has little impact on mean access delay. 


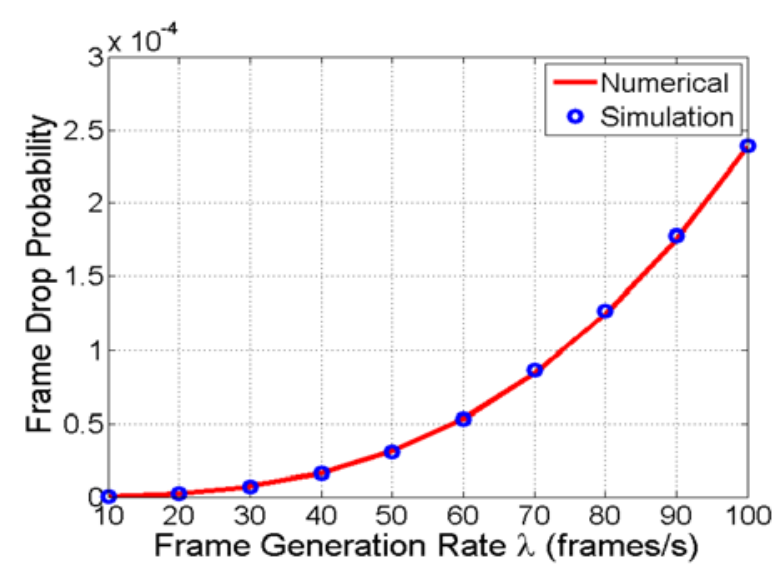

Figure 8. Frame drop probability $\mathrm{P}_{\text {drop }}$ with respect to frame generation rate $\lambda$ of end device

Figure 9 determines the affect of frame size on $\mathrm{P}_{\text {drop. }}$. Here, we consider $2.45 \mathrm{Ghz}$ band with data rate of $250 \mathrm{kbps}$. Packet size varies from 40 bytes to 130 bytes. As can be seen from the figure, as packet size increases, $\mathrm{P}_{\text {drop }}$ decreases. This is because a larger packet size reduces the number of frames generated within a given time duration, which reduces the probability of the radio channel being busy during carrier sensing. Note that the superframe length does not affect $\mathrm{P}_{\text {drop }}$. In addition, it can be seen from figures in section IV that numerical results are very close with simulation results, and this verifies our proposed model.

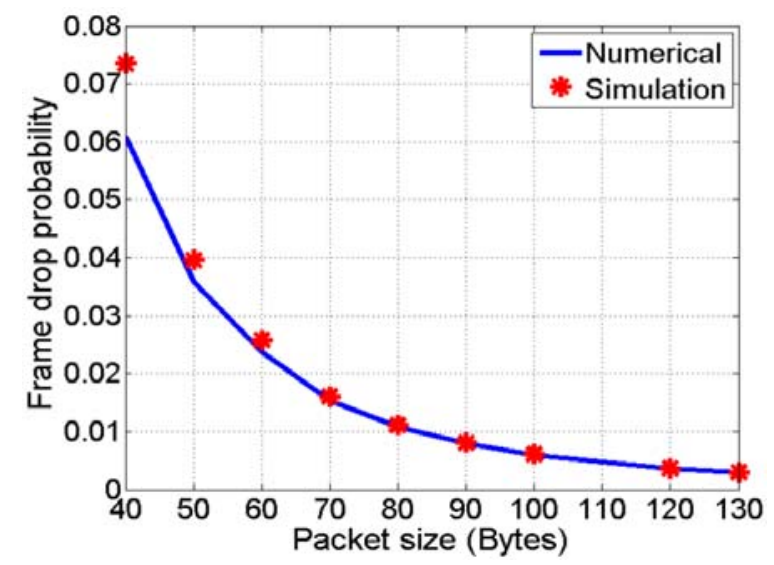

Figure 9. Frame drop probability $\mathrm{P}_{\text {drop }}$ with respect to data frame size.

Finally, Figure 10 presents how MAC average delay depends on Beacon Order (BO). As can be seen from the figure, the delay dynamically increases when $\mathrm{BO}$ increases, in other words, $\mathrm{BO}$ has strong impacts on the delay performance. This is due to the fact that the increased $\mathrm{BO}$ makes superframe longer and thus MAC delay increases as well. The strong dependance of the delay on $\mathrm{BO}$ is due to long beacon interval $\left(\mathrm{BI}=\right.$ aBaseSuperframeDuration $\left.\times 2^{B O}\right)$. 


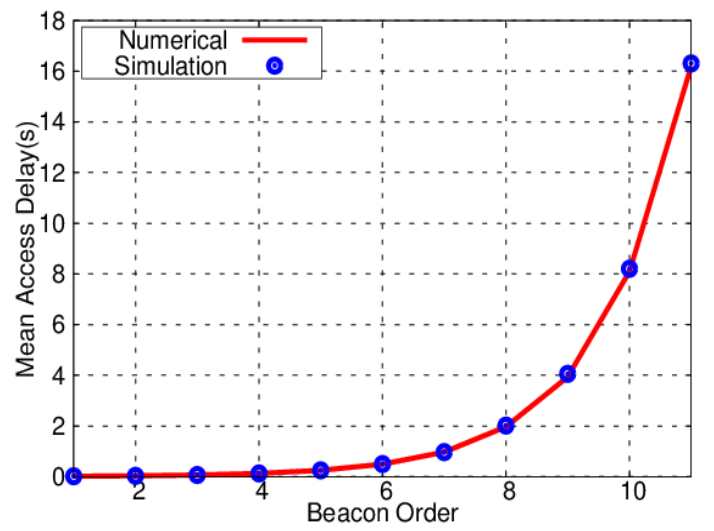

Figure 10. Mean access delay $\mathrm{T}_{D}$ with respect to the beacon order

\section{CONCLUSION}

This paper presents a mathematical analysis of IEEE 802.15.4 beacon-enabled MAC protocol and provides explicit mathematical expressions for power consumption, access delay, and data frame drop probability. The wait for the next beacon frame before backing off in beacon-enabled PAN in fact induces a significant part on access delay. Taking advantage of this operation, we let IEEE 802.15.4 connected devices enter Sleep state to reduce their power consumption. The power model uses different low-power states of the devices and due to those states, power consumption is significantly improved. In addition, the proposed delay model is more completed in comparison to existing research. The mathematical models in this paper allow the application designers to decide on the balance between power consumption and message delay.

\section{REFERENCES}

[1] Part 15.4, "Wireless Medium Access Control (MAC) and Physical Layer (PHY) Specifications for Low-Rate Wireless Personal Area Networks (LR-PANs)," IEEE Std. 802.15.4, 2011.

[2] Ji Z, "Design of an integrated controller based on ZigBee wireless sensor network," TELKOMNIKA Indonesian Journal of Electrical Engineering, vol/issue: 11(8), pp. 4414-4421, 2013.

[3] R. Khadim, et al., "Performance Study of IEEE 802.15. 4 under OPNET Modeler for Wireless Sensor Networks," TELKOMNIKA Indonesian Journal of Electrical Engineering, vol/issue: 16(1), pp. 98-105, 2015.

[4] G. Lu, et al., "Performance evaluation of the ieee 802.15. 4 mac for low-rate low-power wireless networks," IEEE International Conference on Performance, Computing, and Communications, pp. 701-706, 2004.

[5] B. Bougard, et al., "Energy efficiency of the ieee 802.15. 4 standard in dense wireless microsensor networks: Modeling and improvement perspectives," Proc. Design Automation and Test in Europe Conference and Exhibition, pp. 221-234, 2008.

[6] M. Petrova, et al., "Performance study of ieee 802.15. 4 using measurements and simulations," Wireless communications and networking conference, vol. 1, pp. 487-492, 2006.

[7] F. Wang, et al., "Analysis and compare of slotted and unslotted csma in ieee 802.15. 4," Wireless Communications, Networking and Mobile Computing, pp. 1-5, 2009.

[8] G. Anastasi, et al., "A comprehensive analysis of the mac unreliability problem in IEEE 802.15.4 wireless sensor networks," IEEE Transactions on Industrial Informatics, vol/issue: 7(1), pp. 52-65, 2011.

[9] J. Misic, et al., "Performance of ieee 802.15. 4 beacon enabled pan with uplink transmissions in non-saturation modeaccess delay for finite buffers," Proc. First International Conference on Broadband Networks, pp. 416-425, 2004.

[10] S. Pollin, et al., "Performance analysis of slotted carrier sense ieee 802.15. 4 medium access layer," IEEE Trans. On Wireless Comm., vol/issue: 7(9), pp. 3359-3371, 2008.

[11] J. Zhu, et al., "Delay analysis for IEEE 802.15. 4 CSMA/CA scheme with heterogeneous buffered traffic," International Conference on Measuring Technology and Mechatronics Automation (ICMTMA), vol. 1, pp. 835$845,2011$.

[12] G. Bianchi, "Performance analysis of the ieee 802.11 distributed coordination function," IEEE J. Sel. Areas Commun., vol/issue: 18(3), pp. 535-547, 2000.

[13] I. Ramachandran, et al., "Analysis of the contention access period of ieee 802.15. 4 mac," ACM Transactions on Sensor Networks (TOSN), vol/issue: 3(1), pp. 1-29, 2007.

[14] C. Buratti, "Performance analysis of ieee 802.15. 4 beacon-enabled mode," IEEE Transactions on Vehicular Technology, vol/issue: 59(4), pp. 2031-2045, 2010.

[15] B. Latr'e, et al., "Throughput and delay analysis of unslotted ieee 802.15. 4," Journal of Networks, vol/issue: 1(1), pp. 20-28, 2006. 
[16] H. Rasouli, et al., "Adca: Adaptive duty cycle algorithm for energy efficient ieee 802.15. 4 beacon-enabled wireless sensor networks," IEEE Sensor Journal, vol/issue: 14(11), pp. 3893-3902, 2014.

\section{BIOGRAPHIES OF AUTHORS}
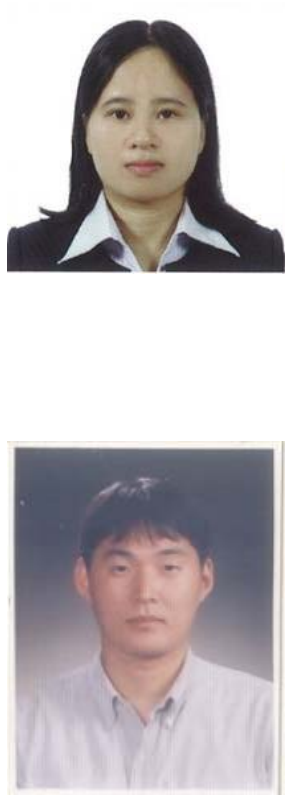

Nga Dinh received the M.S. and Ph. D. degrees in Electrical Engineering from the Korea Institute of Science and Technology (KAIST), Daejeon, Korea, in 2005 and 2009, respectively. From September 2009 to June 2010, she was a Postdoctoral Researcher at the Gwangju Institute of Science and Technology (GIST), Gwangju, Korea. From July 2010 to May 2014, she was a member of technical staff (MTS) at Bell Labs Seoul, Korea. Since August 2014, she works as a senior engineer at IoT Solutions Lab, Software R\&D Center, Samsung Electronics, Seoul, Korea. Her research and development interests include energy efficiency improvement for telecommunication networks, dynamic bandwidth allocations, network resource optimizations, wired/wireless convergence, quality-of-user experience/quality of service (QoE/QoS), wireless connectivity, IoT Solutions, and tinyOS systems.

Sangsoon Lim received Ph. D. degree in the School of Computer Science and Engineering from Seoul National University in 2013. Since October 2013, he works as a senior engineer at Software R\&D Center, Samsung Electronics. His current research interests are in the area of wireless networks including wireless LAN, wireless sensor Networks, cognitive radio networks. 